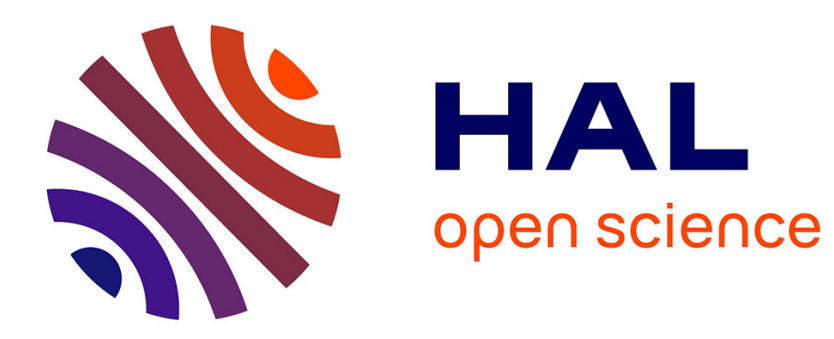

\title{
Shock profiles for the Shallow-water Exner models
}

Christophe Berthon, Benjamin Boutin, Rodolphe Turpault

\section{To cite this version:}

Christophe Berthon, Benjamin Boutin, Rodolphe Turpault. Shock profiles for the Shallow-water Exner models. Advances in Applied Mechanics, 2015, 7 (3), pp.267-294. 10.4208/aamm.2013.m331. hal-01202866

\section{HAL Id: hal-01202866 \\ https://hal.science/hal-01202866}

Submitted on 21 Sep 2015

HAL is a multi-disciplinary open access archive for the deposit and dissemination of scientific research documents, whether they are published or not. The documents may come from teaching and research institutions in France or abroad, or from public or private research centers.
L'archive ouverte pluridisciplinaire HAL, est destinée au dépôt et à la diffusion de documents scientifiques de niveau recherche, publiés ou non, émanant des établissements d'enseignement et de recherche français ou étrangers, des laboratoires publics ou privés. 


\title{
Shock profiles for the Shallow-water Exner models
}

\author{
C. Berthon, B. Boutin and R. Turpault \\ Université de Nantes, LMJL, Nantes, France \\ Université de Rennes 1, IRMAR, Rennes, France
}

\begin{abstract}
This article is devoted to analyze some ambiguities coming from a class of sediment transport models. The models under consideration are governed by the coupling between the shallow-water and the Exner equations. Since the PDE system turns out to be an hyperbolic system in non conservative form, ambiguities may occur as soon as the solution contains shock waves. To enforce a unique definition of the discontinuous solutions, we adopt the path-theory introduced by Dal Maso, LeFLoch and Murat [17]. According to the path choices, we exhibit several shock definitions and we prove that a shock with a constant propagation speed and a given left state may connect an arbitrary right state. As a consequence, additional assumptions (coming from physical considerations or other arguments) must be chosen to enforce a unique definition. Moreover, we show that numerical ambiguities may still exist even when such a choice is made.
\end{abstract}

\section{Introduction}

The numerical simulation of sediment transport is essential in many applications. Indeed, a river flow may carry wide volumes of gravels that seriously modify the river bed. As a consequence, the impact of the sediments transport often cannot be neglected when simulating river flows. For instance, water intakes of some industrial installations may be disturbed by bed river modifications or sediment depositions. Recent sediment transport tools have been derived to perform numerical simulations for bedload. In general, they are based on a suitable coupling between a solid phase model which governs the evolution of the river bed, and a shallow-water model to describe the river flow.

In the present work, we adopt the Exner model [20] to approximate the solid phase. The Exner equation is derived by considering the mass conservation of the solid in the interaction with the river flow. Neglecting dynamical effects, the Exner equation reads:

$$
\partial_{t} z+\partial_{x} \mathcal{Q}(h, u)=0,
$$

where $h>0$ is the water height, $u$ is the height-averaged water speed and $z$ is the height of the river bed. Here, the empirical bedload function $\mathcal{Q}$ is a function of $h$ 
and $u$, which is related to the friction between the water and the sediment that forms the river bed. In practice, many forms of $\mathcal{Q}$ are used depending on the physical setup of the considered problem. The reader is referred to [18, 19, 29, 7] where several bedload formulas are detailed. In this paper, we adopt two distinct bedload functions which are quite representative of the different forms of $\mathcal{Q}$ generally considered for physical simulations. The first one is the simple Grass law [23]:

$$
\mathcal{Q}(h, u)=\varepsilon u^{3},
$$

and the other one, based on the computation of bed stress, was proposed by Nielsen [30]:

$$
\mathcal{Q}(h, u)=\frac{\varepsilon u}{h^{\frac{1}{6}}}\left(\frac{u^{2}}{h^{\frac{1}{3}}}-\tau_{c}\right)_{+} .
$$

In both cases, $\varepsilon$ and $\tau_{c}$ are (usually small) positive parameters and $x_{+}=$ $\max (0, x)$.

The full Exner model is then obtained by coupling the Exner equation (1) to the shallow-water model for taking into account the topography's variations. This full Exner model therefore reads as follows:

$$
\begin{aligned}
\partial_{t} h+\partial_{x} h u & =0, \\
\partial_{t} h u+\partial_{x}\left(h u^{2}+g \frac{h^{2}}{2}\right)+g h \partial_{x} z & =0, \\
\partial_{t} z+\partial_{x} \mathcal{Q}(h, u) & =0,
\end{aligned}
$$

where $g>0$ denotes the gravity constant.

To shorten the notations, let us rewrite the system (4)-(5)-(6) in the following condensed form:

$$
\partial_{t} W+\partial_{x} F(W)+G(W) \partial_{x} W=0
$$

where we have set:

$$
W=\left(\begin{array}{c}
h \\
h u \\
z
\end{array}\right), F(W)=\left(\begin{array}{c}
h u \\
h u^{2}+g \frac{h^{2}}{2} \\
\mathcal{Q}(h, u)
\end{array}\right), G(W)=\left(\begin{array}{ccc}
0 & 0 & 0 \\
0 & 0 & g h \\
0 & 0 & 0
\end{array}\right)
$$

The state vector $W$ is assumed to take values in a convex and open domain $\Omega$ defined by

$$
\Omega=\left\{(h, h u, z) \in \mathbb{R}^{3} ; h>0\right\} .
$$

Let us underline that dry areas are not considered in the present work.

Let us note that the hyperbolicity of the system (4)-(5)-(6) stays an open problem for general forms of the function $\mathcal{Q}$ including (3). In [16], the system (4)(5)-(6) is proved to be strictly hyperbolic when the bedload function is given by the Grass model (2). A sufficient condition for hyperbolicity was also exhibited in [16]. This condition becomes $|u|>6 \sqrt{g h}$ for Nielsen's model but as underlined in [7], the phase state space is certainly larger and no loss of hyperbolicity was reached in simulations, even in extreme configurations. 
Since the models under consideration are hyperbolic, the solution may develop discontinuities in a finite time independently from the smoothness of the initial data. As soon as the system of PDEs writes in conservative form, discontinuous solutions are well understood in a weak sense (for instance see [24, 28]) and the propagation of discontinuities is governed by the well-known RankineHugoniot relations.

Unfortunately, the system (4)-(5)-(6) never recasts in conservative form and a weak interpretation of the solutions cannot be obtained. As widely studied [17, $33,35,3]$, nonconservative products imply ambiguities to define discontinuous solutions. To be more precise, the classical Rankine-Hugoniot relations may no longer be used whenever nonconservative products are involved.

Our main concern in this work is to analyze the impact of the nonconservative term $g h \partial_{x} z$ that appears in (5) since it is a priori not well defined across shocks. Since these products cannot be uniquely defined without additional assumptions, we exhibit the consequences issuing from the lack of a suitable condition for the well-definedness of the nonconservative form of (4)-(5)-(6). Several definitions have been proposed to overcome this ambiguity (see for example $[14,35,17])$. Among them, the theory of Dal Maso, LeFloch and Murat [17] is stable and flexible enough to be used in the numerical context, and to define adapted schemes (see for instance $[32,31,8]$ ).

The loss of uniqueness implied by nonconservative products is all the more problematic in the numerical context since, in general, it is impossible to predict the behavior of the schemes in shocks. Put in other words, the shocks' numerical behavior is distinct from the expected one according to the theory of Dal Maso, LeFloch, Murat (DLM). This failure has been underlined in several contexts $[1,15,26]$. Even though several techniques have been developed to properly take nonconservative terms into account (see for example $[32,22,2,4]$ ), it has been shown in [9] that there are still problems at least in several contexts. In fact, other numerical approaches have been recently introduced to capture the required approximate shock waves, based on viscous profiles regularization $[4,5$, $6]$ or kinetic relations $[11,12]$, which are not available (or prescribed by physics) for the Exner model (4)-(5)-(6). It is worth noticing that numerous recent works still propose or apply numerical schemes for exner models to simulate flows containing shock waves without addressing this issue.

Furthermore, Exner's model has become very popular for the simulation of complex flows involving moving bed and sediment transport. These sophisticated simulations generally include shock discontinuities and our goal is here to illustrate the failure coming from the nonconservative form of the model. The objective of the paper is twofold. On the one hand, we prove that the lack of an additional assumption to describe the shock waves makes the set of admissible discontinuous solutions very large. Formally, almost any discontinuity can be arbitrarily chosen according to a suitable shock definition. On the other hand, we show that the numerical schemes similarly fail. Given an initial discontinuous data, different schemes will capture distinct shock solutions. To conclude, we establish that shock ambiguity is a drastic failure from both analytical and numerical point of view. 
This article is organized as follows. First, the theory of Dal Maso, LeFloch and Murat is recalled and used in the context of the Exner model. In particular, we show several choices of paths which enlighten on the variety of possible definitions for the nonconservative product $g h \partial_{x} z$. Next, the Hugoniot curves for shocks are considered in the case of both Grass and Nielsen forms of $\mathcal{Q}$. Their full expressions are exhibited in the case where the left-state speed $u_{L}=0$, which includes the dam-break problem. Then, several numerical schemes adapted to nonconservative terms are considered. After a short presentation of each of them (including an original one), they are benchmarked over dam-break problems.

\section{Definition of nonconservative products}

In this section, we adopt the theory of Dal Maso, LeFloch and Murat (DLM) [17] to fully characterize shock discontinuities. Our objective is to describe the Hugoniot curves for the Exner model (4)-(5)-(6) according to a fixed path definition. First, the definition of nonconservative products by the DLM theory is recalled. This approach is based on a path definition to be fixed. Several examples are enlightened to show the diversity of the possible definitions and the consequences issuing from this diversity.

\subsection{Resolution of the non-uniqueness: choice of paths}

Let us first recall the DLM theory. We consider terms which write under the nonconservative form $G(W) \partial_{x} W$, where $G$ is a smooth function. If $G$ is the gradient of some function $F$, namely $G(W)=\nabla_{W} F(W)$, then $G(W) \partial_{x} W=$ $\partial_{x} F(W)$ is always defined in the sense of distributions as soon as $W$ is a bounded measurable function.

Otherwise, such a nonconservative product cannot be uniquely defined without additional assumptions. Several definitions were proposed (see for instance $[14,35,17])$. One way to obtain a unique definition was introduced by Dal Maso, LeFloch and Murat in [17]. This technique generalizes the definition of Volpert [35] and provides a stable definition of nonconservative products associated to a choice of path $\Phi$. From now on, let us mention that the prescription of a given path $\Phi$ will yield to a unique definition of the nonconservative product.

Definition 2.1. Let $\Omega$ be a convex and connex subset of $\mathbb{R}^{N}$. A path in $\Omega$ is a local-Lipschitz application

$$
\Phi:[0,1] \times \Omega \times \Omega \rightarrow \Omega,
$$

which satisfies the following properties:

$$
\begin{aligned}
& \text { 1. } \forall\left(W_{L}, W_{R}\right) \in \Omega^{2}, \Phi\left(0, W_{L}, W_{R}\right)=W_{L} \text { and } \Phi\left(1, W_{L}, W_{R}\right)=W_{R} . \\
& \text { 2. } \forall W \in \Omega, \forall \xi \in[0,1], \Phi(\xi, W, W)=W \text {. }
\end{aligned}
$$


3. For every bounded set $\mathcal{O} \in \Omega, \exists K \in \mathbb{R}$ such that $\forall\left(W_{L}^{1}, W_{L}^{2}, W_{R}^{1}, W_{R}^{2}\right) \in$ $\mathcal{O}^{4}$ and for almost all $\xi \in[0,1]$ :

$$
\left\|\frac{\partial \Phi}{\partial \xi}\left(\xi, W_{L}^{2}, W_{R}^{2}\right)-\frac{\partial \Phi}{\partial \xi}\left(\xi, W_{L}^{1}, W_{R}^{1}\right)\right\| \leq K\left(\left\|W_{L}^{2}-W_{L}^{1}\right\|+\left\|W_{R}^{2}-W_{R}^{1}\right\|\right) .
$$

Once a path $\Phi$ is chosen, the nonconservative product is given a sense as a Borel measure. Indeed, if $W \in B V((a, b), \Omega)$ and $G$ a locally bounded Borel function then the nonconservative product of $G(W)$ by $\partial_{x} W$ is denoted

$$
\left[G(W) \partial_{x} W\right]_{\Phi}=\mu
$$

where the Borel measure $\mu$ is characterized by the two following properties:

- If $W$ is continuous on $B$ then:

$$
\mu(B)=\int_{B} G(W(x)) \partial_{x} W(x) d x,
$$

- If $W$ is discontinuous at point $x_{0} \in(a, b)$ where $W\left(x_{0}^{-}\right) \neq W\left(x_{0}^{+}\right)$, then:

$$
\mu\left(\left\{x_{0}\right\}\right)=\int_{0}^{1} G\left(\Phi\left(\xi, W\left(x_{0}^{-}\right), W\left(x_{0}^{+}\right)\right)\right) \frac{\partial \Phi}{\partial \xi}\left(\xi, W\left(x_{0}^{-}\right), W\left(x_{0}^{+}\right)\right) d \xi .
$$

with the notations $\lim _{x_{0}^{-}} W:=W\left(x_{0}^{-}\right)$and $\lim _{x_{0}^{+}} W:=W\left(x_{0}^{+}\right)$,

In the case of the Exner model, the variables are $W=(h, h u, z)^{\top}$ and the nonconservative product follows from:

$$
G(W)=\left(\begin{array}{ccc}
0 & 0 & 0 \\
0 & 0 & g h \\
0 & 0 & 0
\end{array}\right)
$$

Therefore if we denote $\Phi=\left(\Phi_{h}, \Phi_{h u}, \Phi_{z}\right)^{\top}$, the nonconservative term $h \partial_{x} z$ is defined around a discontinuity connecting the states $W_{L}$ and $W_{R}$ by a Dirac mass multiplied by:

$$
\left[h \partial_{x} z\right]_{\Phi}=\int_{0}^{1} \Phi_{h}\left(\xi, W_{L}, W_{R}\right) \frac{\partial \Phi_{z}}{\partial \xi}\left(\xi, W_{L}, W_{R}\right) d \xi
$$

Formally, we will see later that this definition may be viewed as a product of an average of $h$ by the jump of $z$. As a consequence, for the sake of simplicity in the notations, we set:

$$
\left[h \partial_{x} z\right]_{\Phi}=: \bar{h}[z]
$$

where $[z]=\left(z_{R}-z_{L}\right)$ is the jump of $z$ and $\bar{h}$ an average of the water depth to be defined.

Let us point out that, in general, the average $\bar{h}$ may be very sophisticated and may depend on $z$. Several examples will be given in the next section. With 
these notations, the associated generalized Rankine-Hugoniot relations issuing from (4)-(5)-(6) then write:

$$
\begin{aligned}
-\sigma[h]+[h u] & =0, \\
-\sigma[h u]+\left[h u^{2}+g \frac{h^{2}}{2}\right]+g \bar{h}[z] & =0, \\
-\sigma[z]+[\mathcal{Q}(u)] & =0,
\end{aligned}
$$

where $\sigma$ denotes the shock wave velocity.

To conclude this brief presentation of the DLM theory, let us emphasize that the above definition of path may be extended to weaker hypothesis (see for instance [27]) but it is not the purpose of the present work.

\subsection{Examples of paths}

The most common choice of path is the straight line given by:

$$
\Phi\left(\xi, W_{L}, W_{R}\right)=W_{L}+\xi\left(W_{R}-W_{L}\right) .
$$

This path corresponds to Volpert's definition of nonconservative products (see [35]). With this choice, we get:

$$
\begin{aligned}
{\left[h \partial_{x} z\right]_{\Phi} } & =\int_{0}^{1}\left(h_{L}+\xi\left(h_{R}-h_{L}\right)\right)\left(z_{R}-z_{L}\right) d \xi \\
& =\frac{h_{L}+h_{R}}{2}\left(z_{R}-z_{L}\right)
\end{aligned}
$$

so that the $\bar{h}$ term in (10) is simply $\bar{h}=\frac{h_{L}+h_{R}}{2}$, which is nothing but the arithmetic mean value.

In fact, adopting more complex path definitions may give very sophisticated averages $\bar{h}$ eventually depending on the other unknowns. For example, let us suggest the following definition of $\Phi$ :

$$
\Phi\left(\xi, W_{L}, W_{R}\right)=\left(\begin{array}{c}
h_{L}+\xi\left(h_{R}-h_{L}\right) \\
(h u)_{L}+\xi\left((h u)_{R}-(h u)_{L}\right) \\
z_{L}+\xi\left(z_{R}-z_{L}\right)+\xi(1-\xi) \sin \left(z_{R}-z_{L}\right)
\end{array}\right)
$$

to easily get the following mean value for $\bar{h}$ :

$$
\bar{h}=\frac{h_{R}+h_{L}}{2}-\frac{1}{6} \frac{\sin \left(z_{R}-z_{L}\right)}{z_{R}-z_{L}}\left(h_{R}-h_{L}\right) .
$$

It is worth noticing that the above path (14) implies that one may select a path for which $\bar{h}$ does not belong to the interval $\left[\min \left(h_{L}, h_{R}\right), \max \left(h_{L}, h_{R}\right)\right]$, which was already mentioned in [17].

Indeed, we now state that it is also possible to select $\bar{h}$ as any positive mean value of $h_{L}$ and $h_{R}$ through an appropriate choice of path. 
Lemma 2.1. For any admissible $W_{L}$ and $W_{R}$ such that $h_{R} \neq h_{L}$ and any $h^{\star} \in \mathbb{R}_{\star}^{+}$, there exists a path $\Phi$ such that the jump in a discontinuity connecting $W_{L}$ to $W_{R}$ satisfies:

$$
\left[h \partial_{x} z\right]_{\Phi}=h^{\star}[z] .
$$

At this level, it is crucial to notice that the above parameter $h^{\star}>0$ is independent from both left and right states, $W_{L}$ and $W_{R}$. This means that the definition of the nonconservative product (or equivalently the path definition) may drastically modify the Hugoniot curves as described in the next section.

Proof. Let $W_{L}$ and $W_{R}$ be two admissible states such that $h_{R} \neq h_{L}$. In order to simplify the notations, and without loss of generality, we will assume that $h_{R}>h_{L}$.

For $a$ and $\gamma \in(0,1)$ two parameters to be precised later, we introduce the continuous function:

$$
f(\xi)=\left\{\begin{array}{l}
\frac{a}{\gamma} \xi, \text { if } \xi \in(0, \gamma), \\
a \text { if } \xi \in(\gamma, 1-\gamma) \\
1-\frac{1-a}{\gamma}(1-\xi) \text { if } \xi \in(1-\gamma, 1),
\end{array}\right.
$$

and select the following path:

$$
\Phi\left(W_{L}, W_{R}, \xi\right)=\left(\begin{array}{c}
h_{L}+f(\xi)\left(h_{R}-h_{L}\right) \\
(h u)_{L}+\xi\left((h u)_{R}-(h u)_{L}\right) \\
z_{L}+\xi\left(z_{R}-z_{L}\right)
\end{array}\right)
$$

This path is admissible in the sense of Dal Maso, LeFloch and Murat as soon as $\forall \xi \in(0,1), h_{L}+f(\xi)\left(h_{R}-h_{L}\right)>0$ which is equivalent to $h_{L}+a\left(h_{R}-h_{L}\right)>0$ since $h_{L}$ and $h_{R}$ are positive. Furthermore, we have:

$$
\begin{aligned}
& \int_{0}^{1} \Phi_{h}\left(W_{L}, W_{R}, \xi\right) \frac{\partial \Phi_{z}}{\partial \xi}\left(W_{L}, W_{R}, \xi\right) d \xi \\
& =\int_{0}^{1}\left(h_{L}+f(\xi)\left(h_{R}-h_{L}\right)\right)\left(z_{R}-z_{L}\right) d \xi, \\
& =\left(h_{L}+\left(\frac{\gamma}{2}+a(1-\gamma)\right)\left(h_{R}-h_{L}\right)\right)\left(z_{R}-z_{L}\right) .
\end{aligned}
$$

As a consequence, by involving the path (15), the nonconservative product $\left[h \partial_{x} z\right]_{\Phi}$ reads as (10) where $\bar{h}$ is defined by:

$$
\bar{h}=h_{L}+\left(\frac{\gamma}{2}+a(1-\gamma)\right)\left(h_{R}-h_{L}\right),
$$

with $\gamma$ in $(0,1)$ and $h_{L}+a\left(h_{R}-h_{L}\right)>0$.

Now, let us exhibit values of the parameters $a$ and $\gamma$ which allow to recover the expected choice $\bar{h}=h^{\star}$. Indeed, we propose:

$$
\gamma=\frac{1}{2} \min \left(1, \frac{h^{\star}}{\left(h_{L}+h_{R}\right) / 2}\right), \quad a=\frac{y-\frac{\gamma}{2}}{1-\gamma},
$$


where we have set:

$$
y=\frac{h^{\star}-h_{L}}{h_{R}-h_{L}} .
$$

To conclude the proof, we have to verify that $\bar{h}=h^{\star}, \gamma$ stays in $(0,1)$ and $h_{L}+a\left(h_{R}-h_{L}\right)>0$.

By definition of $\bar{h}$, given by (16), the adopted choice of $a$ immediately imposes $\bar{h}=h^{\star}$. Next, since $h^{\star}$ is positive, the definition of $\gamma$ easily ensures $0<\gamma<1$.

Finally, we have:

$$
h_{L}+a\left(h_{R}-h_{L}\right)=\frac{1}{1-\gamma}\left(\frac{h^{\star}}{\left(h_{L}+h_{R}\right) / 2}-\gamma\right) \frac{h_{L}+h_{R}}{2} .
$$

By involving the definition of $\gamma$, we directly obtain the required positiveness of $h_{L}+a\left(h_{R}-h_{L}\right)$ and the proof is achieved.

Let us remark that nothing prevents for using very complicated paths. For instance, one can choose the $a$ coefficient in the proof of lemma 2.1 as a (positive and uniformly bounded) function of $W_{L}$ and $W_{R}$.

To conclude this section, let us underline that the choice of a path is of prime interest to derive some numerical schemes. For instance, in [34], Roe proposed to compute averages as mean values along a well-chosen path. In fact, it is possible to choose paths which are both admissible in the sense of Dal Maso, LeFloch and Murat and suitable to the construction of a Roe scheme. This idea is the basis of path-consistent schemes (see [32] and the description in the following section). In this context of Roe schemes, it is usual to select the straight line path after a suitable change of variables. For instance, using the change of variables $U=(\sqrt{h}, \sqrt{h} u, z)$ and the straight line path for $U$, we obtain: $\bar{h}=\left(h_{L}+\sqrt{h_{L} h_{R}}+h_{R}\right) / 3$. This time, $\bar{h}$ is a mean value of $h_{L}$ and $h_{R}$ but is not a weighted average.

Remark 2.1. As previously mentioned, the choice of path may be related to some physical considerations when they are available. For example, many people consider the straight line path since it is the natural choice to preserve the lakeat-rest ( $h+z$ constant). Obviously, such a physical property may turn out to be unnatural since it eventually allows strong discontinuities in $z$ which won't occur for several types of sediments.

Anyway, even when a physical argument imposes the path, we will see in the last section that the numerical difficulty remains.

\section{Hugoniot curves for the Grass model}

In this paragraph, the Hugoniot curves resulting from the DLM definition of nonconservative products are analyzed in order to characterize the shock solutions inside the phase space (8). More precisely, given a left state $W_{L} \in \Omega$, we consider all the right states $W_{R} \in \Omega$, which can be connected to $W_{L}$ by a shock 
wave. According to the above path definition, we will see that the Hugoniot curve will depend on $\Phi$. Invoking the variety of paths issuing from Lemma 2.1, we will obtain very distinct Hugoniot curves for different path choices.

Here, the Grass model, defined by $\mathcal{Q}(u)=\varepsilon u^{3}$ with $\varepsilon>0$, is adopted. Using this form of bedload function $\mathcal{Q}$ in the generalized Rankine-Hugoniot relations (11)-(12)-(13), we get:

$$
\begin{aligned}
-\sigma[h]+[h u] & =0, \\
-\sigma[h u]+\left[h u^{2}+g \frac{h^{2}}{2}\right]+g \bar{h}[z] & =0, \\
-\sigma[z]+\left[\varepsilon u^{3}\right] & =0 .
\end{aligned}
$$

As a first step, let us point out that no stationary discontinuity may occur. Indeed, assuming $\sigma=0$, from (17), we get $[h u]=0$. Next, from (19), it comes $[u]=0$, which immediately implies, from $[h u]=0$, that $[h]=0$. Finally, we deduce from (18) that $[z]=0$. Therefore, there is no shock with $\sigma=0$.

Let us also remark that if $[h]=0$ then $(17)$ gives $[u]=0$, which implies that $[z]=0$ from (19). Therefore, there is no discontinuous solution as long as $[h]=0$. Similar arguments allow to conclude that there is no discontinuous solution if $[h u]=0$. Since we are only interested in non-trivial shocks, we assume $\sigma \neq 0,[h] \neq 0$ and $[h u] \neq 0$. Now, we are able to exhibit $\sigma$ from (17) as follows:

$$
\sigma=\frac{[h u]}{[h]} .
$$

Inserting the above expression of $\sigma$ in $(19)$ and since $[h u] \neq 0$ in shocks we get:

$$
[z]=\frac{\left[\varepsilon u^{3}\right][h]}{[h u]} .
$$

Then, replacing the above expressions of $[z]$ and $\sigma$ within (18), we obtain:

$$
0=-\frac{[h u]^{2}}{[h]}+\left[h u^{2}+g \frac{h^{2}}{2}\right]+g \bar{h} \frac{\left[\varepsilon u^{3}\right][h]}{[h u]},
$$

which easily rewrites:

$$
0=[h][h u]\left[h u^{2}\right]+g\left[\frac{h^{2}}{2}\right][h][h u]+g \bar{h}[h]^{2}\left[\varepsilon u^{3}\right]-[h u]^{3} .
$$

This last relation is a third order polynomial in $u$ which roots will define the Hugoniot curves in the space $(h, u, z)$. In the general case, these roots have cumbersome values but interestingly, there is a simplification as soon as $u_{L}=0$. Indeed, by involving a vanishing left velocity, the relation (21) reads:

$$
\left(-h^{2} h_{L}+g \varepsilon \bar{h}\left(h-h_{L}\right)^{2}\right) u^{3}+g \frac{h_{L}+h}{2} h\left(h-h_{L}\right)^{2} u=0 .
$$


Now, for the sake of simplicity, we assume that the average parameter $\bar{h}$ only depends on the water height, i.e. $\bar{h}:=\bar{h}\left(h_{L}, h\right)$. Then, as soon as:

$$
\bar{h}<\bar{h}_{0} \quad \text { with } \quad \bar{h}_{0}=\frac{h_{L} h^{2}}{g \varepsilon\left(h-h_{L}\right)^{2}},
$$

the velocity roots, coming from the relation (22), are simply given by:

$$
u=\left\{\begin{array}{l}
0, \\
\pm\left(h-h_{L}\right) \sqrt{\frac{g h\left(h_{L}+h\right)}{2\left(h_{L} h^{2}-g \varepsilon \bar{h}\left(h-h_{L}\right)^{2}\right)}} .
\end{array}\right.
$$

As a consequence, from (20) and (23), we have defined three curves parametrized by $h$ in the phase space $(h, u, z)$ as follows:

$$
\begin{aligned}
& \mathcal{C}_{W_{L}}^{1}=\left\{(h, u, z) \in \mathbb{R}^{3} ; h>0 ; u=-\Psi\left(W_{L}\right) ; z=z_{L}+\varepsilon u^{2} \frac{h-h_{L}}{h}, \bar{h}<\bar{h}_{0}\right\}, \\
& \mathcal{C}_{W_{L}}^{2}=\left\{(h, u, z) \in \mathbb{R}^{3} ; h=h_{L}, u=0, z=z_{L}\right\}, \\
& \mathcal{C}_{W_{L}}^{3}=\left\{(h, u, z) \in \mathbb{R}^{3} ; h>0 ; u=\Psi\left(W_{L}\right) ; z=z_{L}+\varepsilon u^{2} \frac{h-h_{L}}{h}, \bar{h}<\bar{h}_{0}\right\},
\end{aligned}
$$

where we have set:

$$
\Psi\left(W_{L}\right)=\left(h-h_{L}\right) \sqrt{\frac{g h\left(h_{L}+h\right)}{2\left(h_{L} h^{2}-g \varepsilon \bar{h}\left(h-h_{L}\right)^{2}\right)}} .
$$

As expected, these curves define the set of all possible state vectors $W \in \Omega$ that can be connected to $W_{L}=\left(h_{L}, 0, z_{L}\right)^{\top}$ by a discontinuity governed by the generalized Rankine-Hugoniot relations (17)-(18)-(19).

From now on, let us emphasize that the curve $\mathcal{C}_{W_{L}}^{2}$ just defines a trivial stationary shock solution, which is here not of interest.

By considering the whole curves (24), it is well-known that unphysical discontinuities may occur. In order to rule out such non-admissible discontinuous solutions, we adopt the usual Lax condition [24] in a neighborhood of $W_{L}=\left(h_{L}, 0, z_{L}\right)^{\top}$. Since the shock curves are described by considering primitive variables $U=(h, u, z)^{\top}$, we first exhibit the algebraic properties coming from (4)-(5)-(6) in a neighborhood of $W_{L}=\left(h_{L}, 0, z_{L}\right)^{\top}$ but involving primitive variables. The initial system (4)-(5)-(6) easily rewrites as follows:

$$
\partial_{t} U+A(U) \partial_{x} U=0
$$

where we have set

$$
A(U)=\left(\begin{array}{ccc}
u & h & 0 \\
g & u & g \\
\partial_{h} \mathcal{Q} & \partial_{u} \mathcal{Q} & 0
\end{array}\right) .
$$

With $U_{L}=\left(h_{L}, 0, z_{L}\right)^{\top}$ and since $\partial_{h} \mathcal{Q}\left(h_{L}, 0\right)=\partial_{u} \mathcal{Q}\left(h_{L}, 0\right)=0$, the matrix $A\left(U_{L}\right)$ admits three eigenvalues given by $\lambda_{1}=-\sqrt{g h_{L}}, \lambda_{2}=0$ and 
$\lambda_{3}=\sqrt{g h_{L}}$. The associated right eigenvectors are respectively defined by $r_{1}=\left(1, \sqrt{g / h_{L}}, 0\right)^{\top}, r_{2}=(1,0,-1)^{\top}$ and $r_{3}=\left(1,-\sqrt{g / h_{L}}, 0\right)^{\top}$. Using Lax condition in the vicinity of $W_{L}=\left(h_{L}, 0, z_{L}\right)^{\top}$, we immediately deduce that the admissible shock curves associated to a left state at rest are defined by:

$\mathcal{S}_{W_{L}}^{1}=\left\{(h, u, z) \in \mathbb{R}^{3} ; h \geq h_{L} ; u=-\Psi\left(W_{L}\right) ; z=z_{L}+\varepsilon u^{2} \frac{h-h_{L}}{h}, \bar{h}<\bar{h}_{0}\right\}$,

$\mathcal{S}_{W_{L}}^{2}=\left\{(h, u, z) \in \mathbb{R}^{3} ; h=h_{L}, u=0, z=z_{L}\right\}$

$\mathcal{S}_{W_{L}}^{3}=\left\{(h, u, z) \in \mathbb{R}^{3} ; 0<h \leq h_{L} ; u=\Psi\left(W_{L}\right) ; z=z_{L}+\varepsilon u^{2} \frac{h-h_{L}}{h}, \bar{h}<\bar{h}_{0}\right\}$.

The shock curves for 1-shocks and 3-shocks obviously depend on the choice of $\bar{h}$, or equivalently on the choice of the path that defines the nonconservative products. In the following statement, we investigate the variety of shock curves obtained from different values of $\bar{h}$ (according to Lemma 2.1).

Theorem 3.1. Assume that $W_{L}=\left(h_{L}, 0, z_{L}\right)^{\top}$ is given in $\Omega$. Let $\varepsilon>0$ and $W=(h, h u, z)^{\top}$ be given in $\Omega$ such that:

$$
z=z_{L}+\varepsilon u^{2} \frac{h-h_{L}}{h} .
$$

Assume $h>h_{L}$ (resp. $0<h<h_{L}$ ) then there exists $\bar{h}<\bar{h}_{0}$ such that both states $W_{L}$ and $W$ are connected by a 1-shock (resp. a 3-shock) for all $u<$ $-\left(h-h_{L}\right) \sqrt{\frac{g\left(h+h_{L}\right)}{2 h h_{L}}}\left(\right.$ resp. $\left.u>\left(h-h_{L}\right) \sqrt{\frac{g\left(h+h_{L}\right)}{2 h h_{L}}}\right)$.

Proof. Here, we just establish the result for a 1-shock since the proof for a 3 shock follows the same arguments. Let $h>h_{L}$ be given, then from (26) the right velocity, such that the states $W_{L}$ and $W$ are connected by a 1-shock, is given by:

$$
u(\bar{h})=-\left(h-h_{L}\right) \sqrt{\frac{g h\left(h_{L}+h\right)}{2\left(h_{L} h^{2}-g \varepsilon \bar{h}\left(h-h_{L}\right)^{2}\right)}}
$$

with $\bar{h}<\bar{h}_{0}$.

Now, we easily have:

$$
\frac{\partial u}{\partial \bar{h}}(\bar{h})=-\frac{\sqrt{h\left(h+h_{L}\right)}}{2 \sqrt{2 \varepsilon}\left(\bar{h}_{0}-\bar{h}\right) \sqrt{\bar{h}_{0}-\bar{h}}}<0, \forall \bar{h}<\bar{h}_{0} .
$$

Moreover, the following limits are directly obtained:

$$
\begin{aligned}
& \lim _{\bar{h} \rightarrow \bar{h}_{0}} u(\bar{h})=-\infty \\
& \lim _{\bar{h} \rightarrow 0} u(\bar{h})=-\left(h-h_{L}\right) \sqrt{\frac{g\left(h+h_{L}\right)}{2 h h_{L}}} .
\end{aligned}
$$


As a consequence, for all given $u<-\left(h-h_{L}\right) \sqrt{\frac{g\left(h+h_{L}\right)}{2 h h_{L}}}$, there exists $\bar{h}<\bar{h}_{0}$ such that the state vector $U=(h, u, z)$ belongs to the 1 -shock curve. The proof is thus achieved.

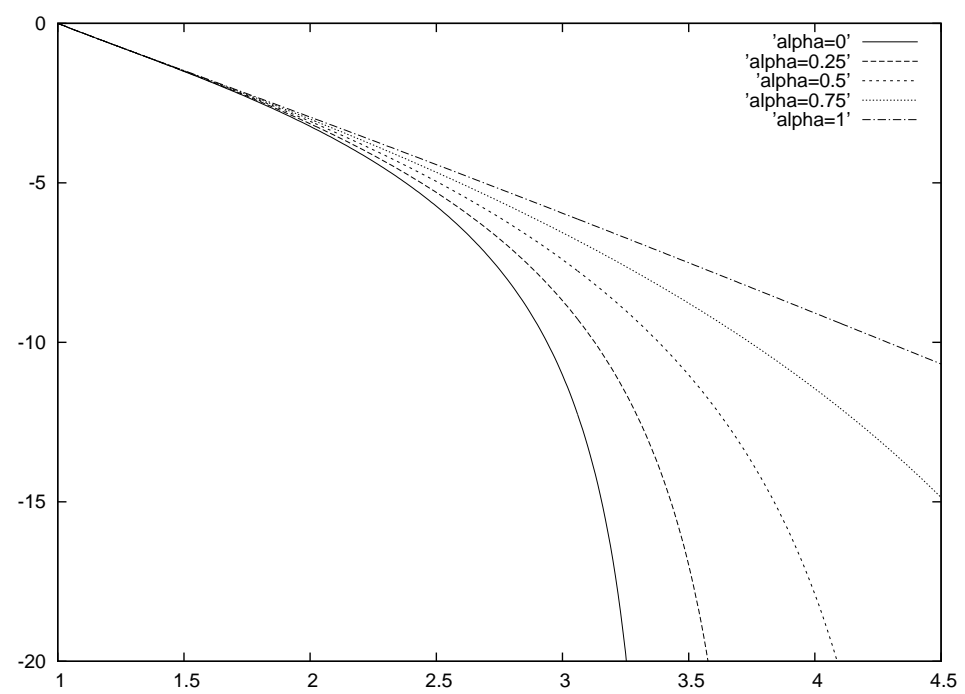

Figure 1: Hugoniot curves from the left state $W_{L}=(1,0,1)^{\top}$ for a 1-shock in the Exner-Grass model: $u$ as a function of $h$ with $\bar{h}=\alpha h_{L}+(1-\alpha) h$ for $\alpha \in[0,1]$.

To illustrate our purpose, Figure 1 shows the Hugoniot curves coming from a 1 -shock with $h_{L}=1$ and $\varepsilon=0.06$. We have imposed $\bar{h}$ as a mean value between $h_{L}$ and $h$ :

$$
\bar{h}=\alpha h_{L}+(1-\alpha) h \quad \text { with } \alpha \in[0,1],
$$

so that Volpert definition of $\bar{h}$ corresponds to $\alpha=1 / 2$. We just recall that this average definition of $\bar{h}$ can be deduced from the path (15) to get (16) as $\bar{h}$ definition, which is nothing but the imposed $\bar{h}$ formula.

Even with these reasonable choice of $\bar{h}$, one can see that there may be important discrepancies between the possible solutions.

\section{Nielsen model}

We turn considering the Nielsen model where the bedload function is defined by:

$$
\mathcal{Q}(u)=\frac{\varepsilon u}{h^{\frac{1}{6}}}\left(\frac{u^{2}}{h^{\frac{1}{3}}}-\tau_{c}\right)_{+},
$$


where $\varepsilon>0$ and $\tau_{c}>0$ are given parameters and $x_{+}=\max (x, 0)$.

Using this form of $\mathcal{Q}$, the Rankine-Hugoniot relations now read:

$$
\begin{aligned}
-\sigma[h]+[h u] & =0, \\
-\sigma[h u]+\left[h u^{2}+g \frac{h^{2}}{2}\right]+g \bar{h}[z] & =0, \\
-\sigma[z]+\left[\frac{\varepsilon u}{h^{\frac{1}{6}}}\left(\frac{u^{2}}{h^{\frac{1}{3}}}-\tau_{c}\right)_{+}\right] & =0 .
\end{aligned}
$$

First, let us notice that no discontinuous solution occurs as soon as $[h]=0$. Indeed, from (29), we get $[u]=0$ and then (30) implies $[z]=0$. Since we exclude trivial shock solutions, we assume $[h] \neq 0$.

The main difficulty comes from the definition of the bedload function, which does not define a bijection. As a consequence, non-trivial discontinuous solutions may satisfy $[h u]=0$ or $\sigma=0$. In fact, this problem can be solved by considering a left state $W_{L}=\left(h_{L}, 0, z_{L}\right)^{\top} \in \Omega$. Indeed, as soon as $u_{L}=0$, the RankineHugoniot relations read:

$$
\begin{aligned}
-\sigma[h]+h u & =0, \\
-\sigma h u+h u^{2}+\left[g \frac{h^{2}}{2}\right]+g \bar{h}[z] & =0, \\
-\sigma[z]+\frac{\varepsilon u}{h^{\frac{1}{6}}}\left(\frac{u^{2}}{h^{\frac{1}{3}}}-\tau_{c}\right)_{+} & =0 .
\end{aligned}
$$

Now, let us assume $h u=0$. Since no dry areas are here considered, we have $h>0$ and then $u=0$. As a consequence, (34) implies $[z]=0$ and next, (33) gives $[h]=0$. Then the solution stays continuous as soon as $h u=0$.

In the same way, if we impose $\sigma=0$, from (32) we get $h u=0$ and once again no discontinuity appears.

As long as we study non-trivial discontinuous solutions issuing from a left state where $u_{L}=0$, we now assume $\sigma \neq 0, u \neq 0$ and $[h] \neq 0$. As a consequence, we can deduce from (32) and (34):

$$
\sigma=\frac{h u}{[h]} \text { and } z=z_{L}+\varepsilon \frac{[h]}{h^{7 / 6}}\left(\frac{u^{2}}{h^{1 / 3}}-\tau_{c}\right)_{+} .
$$

By substituting the above relations satisfied by $[z]$ and $\sigma$ within (33), we get:

$$
g \frac{h+h_{L}}{2}\left(h-h_{L}\right)^{2} h u+g \bar{h}\left(h-h_{L}\right)^{2} \frac{\varepsilon u}{h^{1 / 6}}\left(\frac{u^{2}}{h^{1 / 3}}-\tau_{c}\right)_{+}-h_{L} h^{2} u^{3}=0 .
$$

Let us assume that $\bar{h}$ does not depend on $h$ and $h_{L}$ so that the above equation is easily solved to define $u$ as a function of $h$. Indeed, as soon as $\bar{h} \in\left(0, \bar{h}_{m}\right) \cup$ $\left(\bar{h}_{M},+\infty\right)$ where we have set:

$$
\begin{array}{ll}
\bar{h}_{a}=\frac{h^{\frac{5}{2}} h_{L}}{\varepsilon g\left(h-h_{L}\right)^{2}}, & \bar{h}_{b}=\frac{h^{\frac{7}{6}}\left(h+h_{L}\right)}{2 \varepsilon \tau_{c}}, \\
\bar{h}_{m}=\min \left(\bar{h}_{a}, \bar{h}_{b}\right), & \bar{h}_{M}=\max \left(\bar{h}_{a}, \bar{h}_{b}\right),
\end{array}
$$


the velocity solution reads:

if $u^{2} \geq h^{1 / 3} \tau_{c}$ :

$$
u=\left\{\begin{array}{l}
-\left(h-h_{L}\right) \sqrt{g \frac{2 \varepsilon \bar{h} \tau_{c} h^{\frac{1}{3}}-h^{\frac{3}{2}}\left(h+h_{L}\right)}{2 \varepsilon g \bar{h}\left(h-h_{L}\right)^{2}-2 h^{\frac{5}{2}} h_{L}}}, \\
0, \\
\left(h-h_{L}\right) \sqrt{g \frac{2 \varepsilon \bar{h} \tau_{c} h^{\frac{1}{3}}-h^{\frac{3}{2}}\left(h+h_{L}\right)}{2 \varepsilon g \bar{h}\left(h-h_{L}\right)^{2}-2 h^{\frac{5}{2}} h_{L}}},
\end{array}\right.
$$

if $u^{2}<h^{1 / 3} \tau_{c}$ :

$$
u=\left\{\begin{array}{l}
-\left(h-h_{L}\right) \sqrt{\frac{g\left(h_{L}+h\right)}{2 h h_{L}}}, \\
0, \\
\left(h-h_{L}\right) \sqrt{\frac{g\left(h_{L}+h\right)}{2 h h_{L}}},
\end{array}\right.
$$

To simplify the notations, we introduce the two following functions:

$$
\begin{gathered}
\mathcal{U}^{+}(h)=\left\{\begin{array}{l}
\left(h-h_{L}\right) \sqrt{g \frac{2 \varepsilon \bar{h} \tau_{c} h^{\frac{1}{3}}-h^{\frac{3}{2}}\left(h+h_{L}\right)}{2 \varepsilon g \bar{h}\left(h-h_{L}\right)^{2}-2 h^{\frac{5}{2}} h_{L}}} \text { if } \mathcal{U}^{+}(h)^{2} \geq h^{1 / 3} \tau_{c}, \\
\left(h-h_{L}\right) \sqrt{\frac{g\left(h_{L}+h\right)}{2 h h_{L}}} \text { otherwise, }
\end{array}\right. \\
\mathcal{U}^{+}(h)=\left\{\begin{array}{l}
-\left(h-h_{L}\right) \sqrt{\frac{2 \varepsilon \bar{h} \tau_{c} h^{\frac{1}{3}}-h^{\frac{3}{2}}\left(h+h_{L}\right)}{2 \varepsilon g \bar{h}\left(h-h_{L}\right)^{2}-2 h^{\frac{5}{2}} h_{L}}} \text { if } \mathcal{U}^{+}(h)^{2} \geq h^{1 / 3} \tau_{c}, \\
-\left(h-h_{L}\right) \sqrt{\frac{g\left(h_{L}+h\right)}{2 h h_{L}}} \text { otherwise. }
\end{array}\right.
\end{gathered}
$$

By involving these notations, the solutions of (36) now writes:

$$
u=\left\{\begin{array}{l}
\mathcal{U}^{+}(h) \\
0 \\
\mathcal{U}^{-}(h)
\end{array}\right.
$$

In addition, we set:

$$
\mathcal{Z}^{ \pm}(h)=\left\{\begin{array}{l}
z_{L}+\varepsilon \frac{h-h_{L}}{h^{7 / 6}}\left(\frac{\mathcal{U}^{ \pm}(h)^{2}}{h^{1 / 3}}-\tau_{c}\right)_{+} \text {if } \mathcal{U}^{ \pm}(h)^{2} \geq h^{1 / 3}, \\
z_{L} \quad \text { otherwise },
\end{array}\right.
$$

so that, we note that $z=\mathcal{Z}^{ \pm}(h)$ satisfies the jump condition (35). 
From these definitions we define three continuous curves as follows:

$$
\begin{aligned}
& \mathcal{C}_{W_{L}}^{1}=\left\{(h, u, z)^{\top} \in \mathbb{R}^{3} ; h>0, u=\mathcal{U}^{-}(h), z=\mathcal{Z}^{-}(h), \bar{h} \in\left(0, \bar{h}_{m}\right) \cup\left(\bar{h}_{M},+\infty\right)\right\}, \\
& \mathcal{C}_{W_{L}}^{2}=\left\{(h, u, z)^{\top} \in \mathbb{R}^{3} ; h=h_{L}, u=0, z=z_{L}\right\}, \\
& \mathcal{C}_{W_{L}}^{3}=\left\{(h, u, z)^{\top} \in \mathbb{R}^{3} ; h>0, u=\mathcal{U}^{+}(h), z=\mathcal{Z}^{+}(h), \bar{h} \in\left(0, \bar{h}_{m}\right) \cup\left(\bar{h}_{M},+\infty\right)\right\} .
\end{aligned}
$$

These curves define all the states that can be connected to $W_{L}=\left(h_{L}, 0, z_{L}\right)$ by a discontinuity according to the generalized Rankine-Hugoniot relations (32)(33)-(34). In order to select the admissible part of the curves, we once again adopt the Lax criterion. Hence, we give the algebra satisfied by the matrix $A\left(U_{L}\right)$ defined by (25). Let us note that:

$$
\frac{\partial \mathcal{Q}}{\partial u}(h, u)= \begin{cases}0 & \text { if } u^{2}<h^{1 / 3} \tau_{c}, \\ \frac{\varepsilon}{h^{1 / 6}}\left(3 \frac{u^{2}}{h^{1 / 3}}-\tau_{c}\right) & \text { otherwise }\end{cases}
$$

As a consequence, since dry areas are not considered here, we have $\mathcal{Q}(h, 0)=0$ and $\frac{\partial \mathcal{Q}}{\partial u}(h, 0)=\frac{\partial \mathcal{Q}}{\partial h}(h, 0)=0$. We thus recover the matrix $A(U)$ defined by $(25)$ with the same algebraic properties. Then the admissible shock curves coming from a state at rest are defined as follows:

$$
\begin{aligned}
& \mathcal{S}_{W_{L}}^{1}=\left\{(h, u, z)^{\top} \in \mathbb{R}^{3} ; h \geq h_{L}, u=\mathcal{U}^{-}(h), z=\mathcal{Z}^{-}(h), \bar{h} \in\left(0, \bar{h}_{m}\right) \cup\left(\bar{h}_{M},+\infty\right)\right\}, \\
& \mathcal{S}_{W_{L}}^{2}=\left\{(h, u, z)^{\top} \in \mathbb{R}^{3} ; h=h_{L}, u=0, z=z_{L}\right\}, \\
& \mathcal{S}_{W_{L}}^{3}=\left\{(h, u, z)^{\top} \in \mathbb{R}^{3} ; 0<h \leq h_{L}, u=\mathcal{U}^{+}(h), z=\mathcal{Z}^{+}(h), \bar{h} \in\left(0, \bar{h}_{m}\right) \cup\left(\bar{h}_{M},+\infty\right)\right\} .
\end{aligned}
$$

Now, we study for the behavior of the above shock curves $\mathcal{S}_{W_{L}}^{1}$ and $\mathcal{S}_{W_{L}}^{3}$ according to the definition of $\bar{h}$, or equivalently according to the non-conservative product path definition. Since each shock curve is made of two branches, we first analyze the junction, arising as soon as $u^{2}=h^{1 / 3} \tau_{c}$, of the two parts of the shock curve.

Lemma 4.1. Assume $\bar{h} \neq \bar{h}_{a}$, where $\bar{h}_{a}$ is defined by (37). There exists a unique $h_{\star}>h_{L}$ (resp. $h_{\star}<h_{L}$ ) such that $\mathcal{U}^{-}\left(h_{\star}\right)^{2}=h_{\star}^{\frac{1}{3}} \tau_{c}$ where $\mathcal{U}^{-}$is defined by (39) (resp. $\mathcal{U}^{+}\left(h_{\star}\right)^{2}=h_{\star}^{\frac{1}{3}} \tau_{c}$ where $\mathcal{U}^{+}$is defined by (38)). Furthermore, in all cases, $h_{\star}$ does not depend on the choice of $\bar{h}$.

Proof. By involving the definition of $\mathcal{U}^{ \pm}$, given by (38) and (39), we have:

$$
\mathcal{U}^{ \pm}(h)^{2}-h^{\frac{1}{3}} \tau_{c}=\frac{K(h) N(h)}{D(h)},
$$


where we have set:

$$
\begin{aligned}
& K(h)= \begin{cases}-h^{\frac{3}{2}} & \text { if } \mathcal{U}^{ \pm}(h)^{2} \geq h^{1 / 3} \tau_{c}, \\
1 & \text { otherwise, }\end{cases} \\
& N(h)=-2 h^{\frac{4}{3}} h_{L} \tau_{c}+g\left(h-h_{L}\right)^{2}\left(h+h_{L}\right), \\
& D(h)=\left\{\begin{array}{l}
2 \varepsilon g \bar{h}\left(h-h_{L}\right)^{2}-2 h^{1 / 5} h_{L}, \text { if } \mathcal{U}^{ \pm}(h)^{2} \geq h^{1 / 3} \tau_{c}, \\
2 h h_{L}, \text { otherwise. }
\end{array}\right.
\end{aligned}
$$

Therefore, we remark that $\mathcal{U}^{ \pm}(h)^{2}=h^{\frac{1}{3}} \tau_{c}$ holds either if $h=0$ or $N(h)=0$. Since $h=0$ does not define an admissible state, we now consider the roots of $N(h)=0$.

Introducing the change of variables $\eta=h^{\frac{1}{3}}$, we obtain

$$
N(h)=\mathcal{N}(\eta)=\eta^{9}-h_{L} \eta^{6}-2 \frac{\tau_{c}}{g} h_{L} \eta^{4}-h_{L}^{2} \eta^{3}+h_{L}^{3}
$$

Straightforward computations give:

$$
\mathcal{N}^{\prime}(\eta)=\eta^{2} p(\eta)
$$

where:

$$
p(\eta)=9 \eta^{6}-6 h_{L} \eta^{3}-8 \frac{\tau_{c}}{g} \eta h_{L}-3 h_{L}^{2} .
$$

Moreover, we easily have:

$$
\begin{aligned}
& p^{\prime}(\eta)=54 \eta^{5}-18 h_{L} \eta^{2}-8 \frac{\tau_{c}}{g} h_{L}, \\
& p^{\prime \prime}(\eta)=18 \eta\left(15 \eta^{3}-2 h_{L}\right) .
\end{aligned}
$$

The roots of $p^{\prime \prime}$ are 0 and $\eta_{1}=\left(\frac{2 h_{L}}{15}\right)^{\frac{1}{3}}$, therefore $p^{\prime \prime}<0$ if $\eta \in\left[0, \eta_{1}\right]$ and $p^{\prime \prime}>0$ if $\eta \in\left[\eta_{1},+\infty\right)$. As a consequence, $p^{\prime}$ is decreasing for $\eta \in\left[0, \eta_{1}\right]$ and increasing for $\eta \in\left[\eta_{1},+\infty\right)$. Since $p^{\prime}(0)<0$, we immediately deduce that there exists $\eta_{2}>\eta_{1}$ such that $p^{\prime}<0$ if $\eta \in\left[0, \eta_{2}\right]$ and $p^{\prime}>0$ if $\eta \in\left[\eta_{2},+\infty\right)$. The same arguments may be applied to $p$ since $p(0)<0$. Then there exists $\eta_{3}>\eta_{2}$ such that $p<0$ if $\eta \in\left[0, \eta_{3}\right]$ and $p>0$ if $\eta \in\left[\eta_{3},+\infty\right)$.

Finally, since $\mathcal{N}^{\prime}(\eta)=\eta^{2} p(\eta)$, we deduce that $\mathcal{N}$ is decreasing if $\eta \in\left[0, \eta_{3}\right]$ and increasing if $\eta \in\left[\eta_{3},+\infty\right)$. Now, we notice that $\mathcal{N}\left(h_{L}^{1 / 3}\right)=N\left(h_{L}\right)=$ $-2 \frac{\tau_{c}}{g} h_{L}^{\frac{7}{3}}<0$, which implies that the equation $\mathcal{N}(\eta)=0$ admits a unique solution $\eta^{\star}>h_{L}^{1 / 3}$. Therefore, the equation $N(h)=0$ has only one root $h_{\star}$ larger than $h_{L}$, and the proof is completed.

To conclude the presentation of the Hugoniot curves for the Nielsen model, we now establish that a large variety of shock curves can be reached by involving suitable path definitions, or equivalently by involving suitable $\bar{h}$ definitions according to Lemma 2.1 . 
Theorem 4.1. Assume $W_{L} \in \Omega$ be given such that $u_{L}=0$. Let $\varepsilon>0, \tau_{c}>0$ be two given constants. Let $W=(h, h u, z)^{\top}$ be an admissible state in $\Omega$ such that $h>h_{L}$ (resp. $0<h<h_{L}$ ) and $z=\mathcal{Z}^{-}(h)$ (resp. $z=\mathcal{Z}^{+}(h)$ ) where $\mathcal{Z}^{ \pm}$ are defined by $(40)$.

- If $h \leq h_{\star}$ (resp. $h \geq h_{\star}$ ), where $h_{\star}$ is defined Lemma 4.1, then the 1-shock curve (resp. the 3-shock curve) is uniquely defined independently from the choice of $\bar{h}$.

- If $h>h_{\star}$ (resp. $h<h_{\star}$ ) then there exists $\bar{h} \in\left(0, \bar{h}_{m}\right) \cup\left(\bar{h}_{M},+\infty\right)$, where $\bar{h}_{m}$ and $\bar{h}_{M}$ are defined by (37), such that the state $W_{L}=\left(h_{L}, 0, z_{L}\right)^{\top}$ and $W$ are connected by a 1-shock (resp. a 3-shock) for all $u \in(-\infty,-\tilde{h}) \cup$ $\left(-\sqrt{\tau_{c} h^{1 / 3}}, 0\right)\left(\right.$ resp. $\left.u \in\left(0, \sqrt{\tau_{c} h^{1 / 3}}\right) \cup(\tilde{h},+\infty)\right)$ where we have set:

$$
\tilde{h}=\left(h-h_{L}\right) \sqrt{g \frac{h+h_{L}}{2 h h_{L}}} .
$$

Proof. We just consider 1-shock solutions. The proof turns out to be similar considering 3 -shock solutions. Across a 1-shock connecting a left state at rest, the velocity is given by $u=\mathcal{U}^{-}(h)$ where $\mathcal{U}^{-}$is given by (39). Now, by involving the definition of $h_{\star}$, the function $\mathcal{U}^{-}$rewrites:

$$
\mathcal{U}^{-}(h)=\left\{\begin{array}{l}
-\left(h-h_{L}\right) \sqrt{g \frac{2 \varepsilon \bar{h} \tau_{c} h^{\frac{1}{3}}-h^{\frac{3}{2}}\left(h+h_{L}\right)}{2 \varepsilon g \bar{h}\left(h-h_{L}\right)^{2}-2 h^{\frac{5}{2}} h_{L}}}, \quad \text { if } h>h_{\star}, \\
-\left(h-h_{L}\right) \sqrt{\frac{g\left(h_{L}+h\right)}{2 h h_{L}}}, \quad \text { otherwise. }
\end{array}\right.
$$

Similarly, the topography function $z$ is given by $z=\mathcal{Z}^{-}(h)$ where $\mathcal{Z}^{-}$reads:

$$
\mathcal{Z}^{-}(h)= \begin{cases}z_{L}+\varepsilon \frac{h-h_{L}}{h^{7 / 6}}\left(\frac{\mathcal{U}^{-}(h)^{2}}{h^{1 / 3}}-\tau_{c}\right)_{+} & \text {if } h>h_{\star}, \\ z_{L} \quad \text { otherwise. } & \end{cases}
$$

We immediately remark that $u=\mathcal{U}^{-}(h)$ and $z=\mathcal{Z}^{-}(h)$ are uniquely defined (independently from $\bar{h}$ ) as long as $0<h \leq h_{\star}$.

Now, we turn considering $h>h_{\star}$. To characterized the 1-shock solution, we have to study for the function $\mathcal{U}^{-}$since the definition of $\mathcal{Z}^{-}$just involves $\mathcal{U}^{-}$, which now writes

$$
\mathcal{U}^{-}(h)=-\left(h-h_{L}\right) \sqrt{g} \sqrt{\frac{n(\bar{h})}{d(\bar{h})}},
$$

where we have set:

$$
\begin{aligned}
& n(\bar{h})=2 \varepsilon \bar{h} \tau_{c} H^{1 / 3}-h^{2 / 3}\left(h+h_{L}\right), \\
& d(\bar{h})=2 \varepsilon g \bar{h}\left(h-h_{L}\right)^{2}-2 h^{5 / 2} h_{L} .
\end{aligned}
$$


We recall that $d(\bar{h}) \neq 0$ as long as $\bar{h} \neq \bar{h}_{a}$ where $\bar{h}_{a}$ is given by (37), and $\frac{n(\bar{h})}{d(\bar{h})}>0$ as long as $\bar{h} \in\left(0, \bar{h}_{m}\right) \cup\left(\bar{h}_{M},+\infty\right)$.

Straightforward computations yield to the following sequence of equalities:

$$
\begin{aligned}
\partial_{\bar{h}} \mathcal{U}^{-} & =-\frac{\left(h-h_{L}\right) \sqrt{g}}{2 \sqrt{n(\bar{h}) / d(\bar{h})}} \frac{\partial_{\bar{h}} n(\bar{h}) d(\bar{h})-n(\bar{h}) \partial_{\bar{h}} d(\bar{h})}{d(\bar{h})^{2}}, \\
& =-\frac{\left(h-h_{L}\right) \sqrt{g} h^{\frac{3}{2}}}{\sqrt{n(\bar{h}) / d(\bar{h})} d(\bar{h})^{2}}\left(-2 \tau_{c} h_{L} h^{\frac{4}{3}}+g\left(h-h_{L}\right)^{2}\left(h+h_{L}\right)\right), \\
& =-\frac{\left(h-h_{L}\right) \sqrt{g} h^{\frac{3}{2}} N(h)}{\sqrt{n(\bar{h}) d(\bar{h})}},
\end{aligned}
$$

where the function $N$ is defined by (44) and stays positive for all $h>h_{\star}$. As a consequence, we get $\partial_{\bar{h}} \mathcal{U}^{-}<0$ for all $h>h_{\star}$. Therefore, for $h>h_{\star}$ fixed, $\mathcal{U}^{-}$ is a decreasing function of $\bar{h} \in\left(0, \bar{h}_{m}\right) \cup\left(\bar{h}_{M},+\infty\right)$. Moreover, An immediate consequence of the definitions of $\bar{h}_{a}$ and $\bar{h}_{b}$, defined by (37), gives for all fixed $h>h_{\star}$ :

$$
\lim _{\bar{h} \rightarrow \bar{h}_{a}} \mathcal{U}^{-}=-\infty, \lim _{\bar{h} \rightarrow \bar{h}_{b}} \mathcal{U}^{-}=0 .
$$

Since for $h>h_{\star}, \mathcal{U}^{-}$is a decreasing function of $\bar{h}$ in $\left(0, \min \left(\bar{h}_{a}, \bar{h}_{b}\right)\right) \cup\left(\max \left(\bar{h}_{a}, \bar{h}_{b}\right),+\infty\right)$, then necessarily $\bar{h}_{a}<\bar{h}_{b}$.

The proof directly follows from the following limits:

$$
\begin{aligned}
& \lim _{h \rightarrow 0} \mathcal{U}^{-}=-\left(h-h_{L}\right) \sqrt{g \frac{h+h_{L}}{2 h h_{L}}} \\
& \text { and } \lim _{\bar{h} \rightarrow+\infty} \mathcal{U}^{-}=-\sqrt{\tau_{c} h^{1 / 3}} .
\end{aligned}
$$

To illustrate our purpose, the function $\mathcal{U}^{-}$versus $\bar{h}$ with the parameters $h=1.5, h_{L}=1, \tau_{c}=1$ and $\varepsilon=0.06$ is shown on Figure 2. Here $\tau_{c}$ was chosen large enough for the sake of presentation although it is usually smaller in applications. In realistic simulations, since $\bar{h}_{b}$ is very large, numerical schemes are expected to involve values of $\bar{h}$ within the interval $\left(0, \bar{h}_{a}\right)$.

In a similar fashion, Figure 3 shows the curve of a 1-shock, this time as a function of $h$ with $h_{L}=1, \tau_{c}=0.05$ and $\varepsilon=0.06$. For this picture, $\bar{h}$ once again was taken as a mean value between $h_{L}$ and $h$, that is $\bar{h}=\alpha h_{L}+(1-\alpha) h$ for $\alpha \in[0,1]$, so that Volpert definition of $\bar{h}$ corresponds to $\alpha=1 / 2$.

As for Grass model, there are still important discrepancies between the possible solutions even with reasonable choices of parameters and $\bar{h}$. 


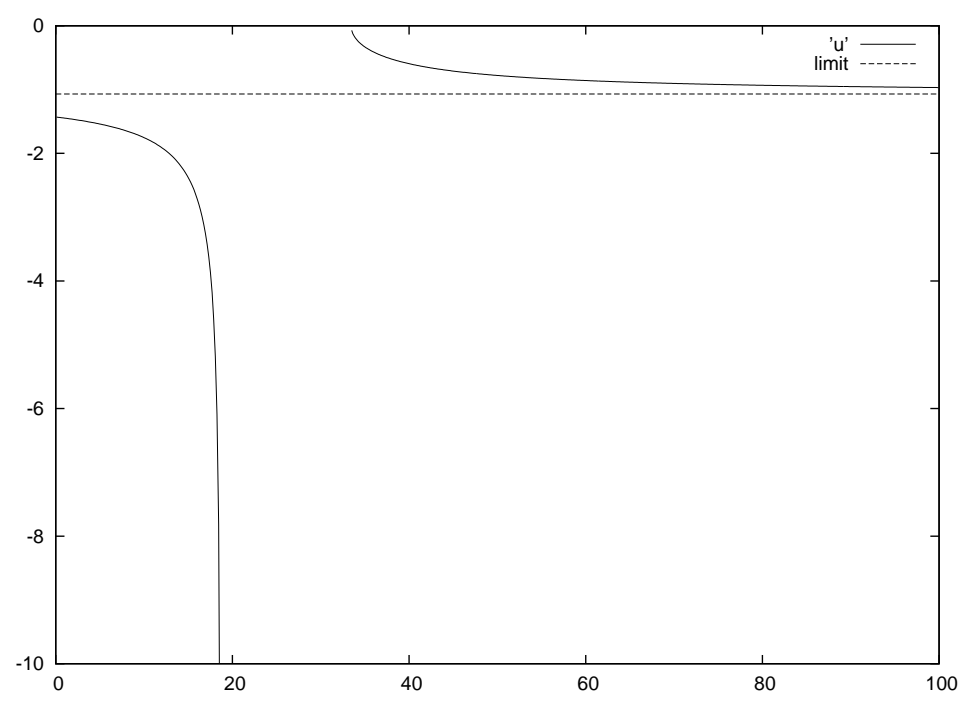

Figure 2: Hugoniot curves for a 1-shock in the Exner-Nielsen model: $u$ as a function of $\bar{h}$ and the limit value $-\sqrt{\tau_{c} h^{\frac{1}{3}}}$.

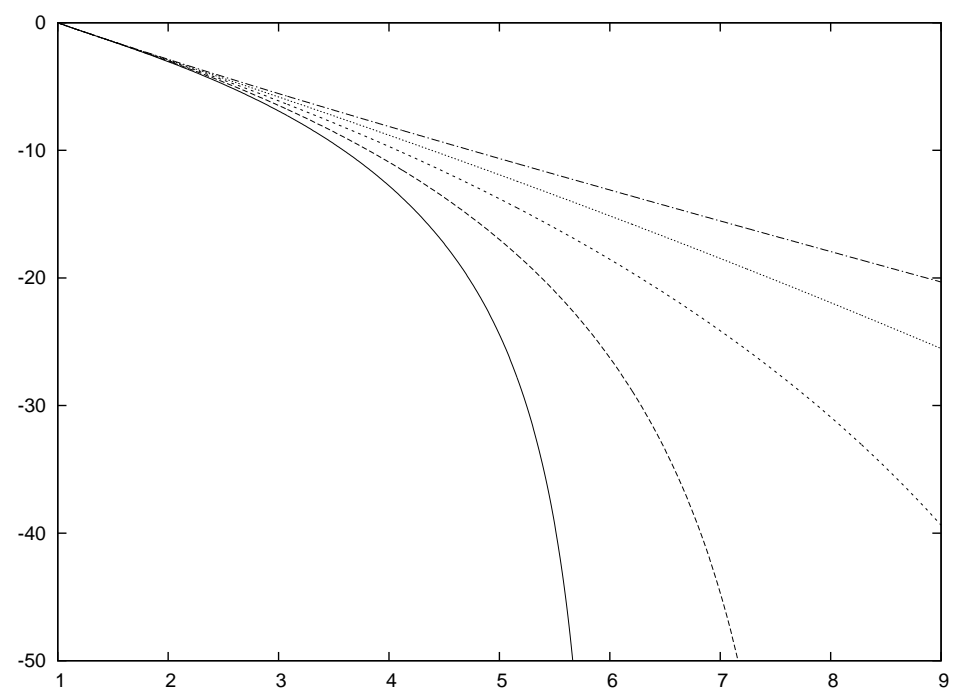

Figure 3: Hugoniot curves for a 1-shock in the Exner-Nielsen model: $u$ as a function of $h$ with the choice $\bar{h}=\alpha h_{L}+(1-\alpha) h$ for $\alpha=$ (bottom to top) $0,0.25,0.5,0.75$ and 1 .

\section{$5 \quad$ Numerical results}

This section concerns four numerical techniques for taking into account nonconservative terms which allow to design adapted numerical schemes. That is to be 
the so-called path-consistent schemes developed by Parès and Castro [31, 32], a scheme proposed by Ghidaglia, Kumbaro and LeCoq [22], a relaxation scheme specifically designed for the Exner system [2] and a modified HLL scheme constructed for the sake of this article.

Each technique is briefly described, then they are all applied to dam-break problems in order to investigate their behavior in shocks.

\subsection{Short description of the schemes}

Path-consistent scheme Path-consistent schemes were introduced in [31] (see also [32]) and extended to take into account more complex physics (see for instance [8]).

These schemes read as a natural extension of the well-known Roe scheme, adapted to the nonconservative context: a suitable path is selected in order to define the Roe matrix for the conservative part. Then, the same path is also used for the definition of the nonconservative terms.

Indeed, Roe proposed in [34] a convenient way to obtain suitable Roe matrices to derive the numerical scheme. It consists in selecting a path and to compute the averages by integrating along this path. In practice, the path is often the straight line path for a new set of variables. For instance, Roe used the change of variables $Z=\rho^{-\frac{1}{2}} W$ to approximate Euler equations.

The idea of Castro and Parès is to compute the Roe matrix of their numerical schemes by using the same path that defines the nonconservative product. In the end, the expression of the scheme is similar to any Roe scheme: first, a suitable way to compute Roe averages, which is denoted $\mathcal{A}\left(W_{1}, W_{2}\right)$, is selected. This Roe average has to be computed over a path which is admissible both from the Roe and Dal Maso-LeFloch-Murat theories.

Then we note $\mathcal{A}_{i+\frac{1}{2}}=\mathcal{A}\left(W_{i}^{n}, W_{i+1}^{n}\right)$. Since $\mathcal{A}_{i+\frac{1}{2}}$ is a Roe matrix, it is diagonalizable i.e. $\mathcal{A}_{i+\frac{1}{2}}=P_{i+\frac{1}{2}} \Lambda_{i+\frac{1}{2}} P_{i+\frac{1}{2}}^{-1}$ where $\Lambda_{i+\frac{1}{2}}$ is the diagonal matrix composed of the eigenvalues of $\mathcal{A}_{i+\frac{1}{2}}$. Now, setting $\mathcal{A}_{i+\frac{1}{2}}^{ \pm}=P_{i+\frac{1}{2}} \Lambda_{i+\frac{1}{2}}^{ \pm} P_{i+\frac{1}{2}}^{-1}$ where

$$
\Lambda_{i+\frac{1}{2}}^{ \pm}=\left(\begin{array}{ccc}
\lambda_{1}^{ \pm} & & 0 \\
& \ddots & \\
0 & & \lambda_{N}^{ \pm}
\end{array}\right),
$$

and $a^{ \pm}$respectively represents the positive and negative parts of $a$. The scheme finally reads:

$$
W_{i}^{n+1}=W_{i}^{n}-\frac{\Delta t}{\Delta x}\left(\mathcal{A}_{i+\frac{1}{2}}^{-}\left(W_{i+1}^{n}-W_{i}^{n}\right)+\mathcal{A}_{i-\frac{1}{2}}^{+}\left(W_{i}^{n}-W_{i-1}^{n}\right)\right),
$$

In practice, the straight line path is often considered for the sake of simplicity in the computations. For the numerical simulations performed in this paper, the straight lines path for the variables $(\sqrt{h}, \sqrt{h} u, z)$ was considered as in [7]. 
The scheme of Ghidaglia, Kumbaro and Le Coq This scheme was introduced in [22]. In the conservative context, it is built as follows. The formal conservation law:

$$
\partial_{t} W+\partial_{x} f(W)=0
$$

is approximated around each interface of the mesh, setting $A(W)=\partial_{W} F(W)$, by:

$$
\partial_{t} W+A\left(\mu_{i+\frac{1}{2}}\right) \partial_{x} W=0,
$$

where $\mu_{i+\frac{1}{2}}$ is a mean value of $W$ in the neighborhood of the interface $x_{i+\frac{1}{2}}$. The numerical flux at $x_{i+\frac{1}{2}}$ is then chosen to be:

$$
\begin{aligned}
\mathcal{F}_{i+\frac{1}{2}}^{n} & =\sum_{\lambda_{k}<0}\left[l_{k} F\left(W_{i}^{n}\right)\right] r_{k}+\sum_{\lambda_{k}>0}\left[l_{k} F\left(W_{i+1}^{n}\right)\right] r_{k} \\
& +\sum_{\lambda_{k}=0}\left[\frac{l_{k}}{2}\left(F\left(W_{i}^{n}\right)+F\left(W_{i+1}^{n}\right)\right)\right] r_{k},
\end{aligned}
$$

where $\lambda_{k}, l_{k}$ and $r_{k}$ are respectively the eigenvalues, left and right eigenvectors of $A\left(\mu_{i+\frac{1}{2}}\right)$. The overall scheme writes then:

$$
W_{i}^{n+1}=W_{i}^{n}-\frac{\Delta t}{\Delta x}\left(\mathcal{F}_{i+\frac{1}{2}}^{n}-\mathcal{F}_{i-\frac{1}{2}}^{n}\right) .
$$

Now, let us consider a nonconservative system in the following form:

$$
\partial_{t} W+\partial_{x} f(W)+C(W) \partial_{x} W=0 .
$$

We set:

$$
J(W)=\nabla f(W) \quad \text { and } \quad E(W)=C(W) J(W)^{-1},
$$

as long as the matrix $J$ is invertible, to introduce the following numerical discretization:

$$
W_{i}^{n+1}=W_{i}^{n}-\frac{\Delta t}{\Delta x}\left[I+E\left(W_{i}^{n}\right)\right]\left(\mathcal{F}_{i+\frac{1}{2}}^{n}-\mathcal{F}_{i-\frac{1}{2}}^{n}\right),
$$

where the $\mathcal{F}_{i+\frac{1}{2}}^{n}$ 's are defined by (45). The choice of both matrices $J$ and $E$ is not unique since the following relation holds:

$$
\partial_{x} F(W)+C(W) \partial_{x} W=\partial_{x}(F(W)+G(W))+\left(C(W)-\nabla_{W} G(W)\right) \partial_{x} W .
$$

Put in other words, the choice of $J$ and $E$ is related to the choice of a path to define the nonconservative product.

In the case of Exner's model with Grass law, one would like to choose:

$$
J(W)=\left(\begin{array}{ccc}
0 & 1 & 0 \\
-u^{2}+g h & 2 u & 0 \\
-3 \varepsilon \frac{u^{3}}{h} & 3 \varepsilon \frac{u^{2}}{h} & 0
\end{array}\right)
$$


which is the Jacobian matrix of the conservative terms. This is not possible however since this matrix $J$ is obviously singular. To derive the required scheme, we adopt the following definition for $J$ :

$$
J(W)=\left(\begin{array}{ccc}
0 & 1 & 0 \\
-u^{2}+g h & 2 u & 0 \\
-3 \varepsilon \frac{u^{3}}{h} & 3 \varepsilon \frac{u^{2}}{h} & 1
\end{array}\right) \text { and } C(W)=\left(\begin{array}{ccc}
0 & 0 & 0 \\
0 & 0 & g h \\
0 & 0 & -1
\end{array}\right) .
$$

Let us note that Ghidaglia, Kumbaro and Le Coq developed an implicit version of their numerical scheme in order to avoid the stability problems which may arise in the explicit case. Only the explicit scheme was considered in this paragraph.

A relaxation scheme This scheme was introduced in [2]. Its is based on the following relaxation model:

$$
\begin{aligned}
\partial_{t} h+\partial_{x} h u & =0, \\
\partial_{t} h u+\partial_{x}\left(h u^{2}+\Pi\right)+g h \partial_{x} z & =0, \\
\partial_{t} \Pi+u \partial_{x} \Pi+\frac{a^{2}}{h} \partial_{x} u & =\frac{1}{\lambda}\left(\frac{g h^{2}}{2}-\Pi\right), \\
\partial_{t} z+\partial_{x} \Omega & =0, \\
\partial_{t} \Omega+\left(\frac{b^{2}}{h^{2}}-u^{2}\right) \partial_{x} z+2 u \partial_{x} \Omega & =\frac{1}{\lambda}(\mathcal{Q}(h, u)-\Omega),
\end{aligned}
$$

where $a$ and $b$ are parameters which are chosen to ensure the stability of the numerical scheme.

The resulting numerical scheme is composed of two steps. The first one is dedicated to the transport part while the second step is devoted to deal with the stiff source term.

During the first step, the relaxation unknowns are evolved by considering the homogeneous system issuing from the relaxation model (46). In fact, the extracted homogeneous system turns out to be fully linearly degenerated and the associated Riemann problem is easily solvable. As a consequence, this first step consists in a classical Godunov scheme to update the relaxation unknowns.

The second step simply consists in a projection to the equilibrium map that corresponds to all admissible states $(h, u, \Pi, z, \Omega)$ such that $\Pi=g h^{2} / 2$ and $\Omega=\mathcal{Q}(h, u)$. In the end, the whole scheme can be written in order to only update $h, h u$ and $z$.

Finally, the scheme was shown to be robust under Whitham subcharacteristic type conditions taking the form $a \geq h \sqrt{g h}$ and $b \geq \sqrt{(h u)^{2}+g h^{2} \partial_{u} \mathcal{Q}}$. Indeed, these parameters $a$ and $b$ control the numerical diffusion of the scheme.

HLL type scheme This scheme is a modification of the classical Rusanov scheme using the consistency with the integral form proposed in the paper of Harten, Lax and Van Leer [25] in order to handle nonconservative terms. This 
idea is related to the ones proposed in [13, 21].After [25], the numerical scheme comes from a derivation of a suitable approximate Riemann solver. Here, we adopt an approximate Riemann solver made of one intermediate state $W_{\star}$. Following [25] (see also [21]), the condition of compatibility with the integral form gives the following relations:

$$
\begin{aligned}
& -b\left(h_{\star}-h_{L}\right)+b\left(h_{R}-h_{\star}\right)=-\left(F^{h}\left(W_{R}\right)-F^{h}\left(W_{L}\right)\right), \\
& -b\left((h u)_{\star}-(h u)_{L}\right)+b\left((h u)_{R}-(h u)_{\star}\right) \\
& =-\left(F^{h u}\left(W_{R}\right)-F^{h u}\left(W_{L}\right)\right)-\frac{1}{\Delta t} \int_{t^{n}}^{t^{n+1}} \int_{x_{i-\frac{1}{2}}}^{x_{i+\frac{1}{2}}} \Phi_{h} \frac{\partial \Phi_{z}}{\partial \xi}(\xi) d \xi d t, \\
& -b\left(z_{\star}-z_{L}\right)+b\left(z_{R}-z_{\star}\right)=-\left(F^{z}\left(W_{R}\right)-F^{z}\left(W_{L}\right)\right),
\end{aligned}
$$

where $b>0$ stands for a numerical acoustic speed.

As a consequence, we easily deduce the value of $W_{\star}$ given by:

$$
\begin{aligned}
h_{\star} & =\frac{h_{L}+h_{R}}{2}-\frac{F^{h}\left(W_{R}\right)-F^{h}\left(W_{L}\right)}{2 b}, \\
(h u)_{\star} & =\frac{(h u)_{L}+(h u)_{R}}{2}-\frac{F^{h u}\left(W_{R}\right)-F^{h u}\left(W_{L}\right)}{2 b}-\frac{\Delta x}{2 b} \bar{h}\left(z_{R}-z_{L}\right), \\
z_{\star} & =\frac{z_{L}+z_{R}}{2}-\frac{F^{z}\left(W_{R}\right)-F^{z}\left(W_{L}\right)}{2 b} .
\end{aligned}
$$

Once the intermediate state known, the scheme is obtained as usual by integrating the approximate Riemann solution over each cell. We thus obtain:

$$
\begin{aligned}
h_{i}^{n+1} & =h_{i}^{n}-\frac{\Delta t}{\Delta x}\left(\mathcal{F}_{i \frac{1}{2}}^{h}-\mathcal{F}_{i-\frac{1}{2}}^{h}\right), \\
(h u)_{i}^{n+1} & =(h u)_{i}^{n}-\frac{\Delta t}{\Delta x}\left(\mathcal{F}_{i \frac{1}{2}}^{h u}-\mathcal{F}_{i-\frac{1}{2}}^{h u}\right)-\frac{\Delta t}{2}\left(\bar{h}_{i+\frac{1}{2}}\left(z_{i+1}-z_{i}\right)+\bar{h}_{i-\frac{1}{2}}\left(z_{i}-z_{i-1}\right)\right), \\
z_{i}^{n+1} & =z_{i}^{n}-\frac{\Delta t}{\Delta x}\left(\mathcal{F}_{i \frac{1}{2}}^{z}-\mathcal{F}_{i-\frac{1}{2}}^{z}\right),
\end{aligned}
$$

where the involved numerical flux functions are given by the usual HLL flux function associated with the exact flux function (7).

Let us point out that this scheme obviously depends on the choice of path made to define $\bar{h}$. In numerical simulations, the straight line path is used unless otherwise specified.

\subsection{Numerical Hugoniot curves}

The four numerical schemes are now used to compute the solutions of Riemann problems corresponding to $h_{L}=0$ and $u_{L}=0$ on a domain of length 1 meshed with 2000 cells. The results are compared between one another and with the theoretical Hugoniot curves obtained in previous sections. For these comparisons, $\bar{h}$ is chosen to be a mean value of $h$ and $h_{L}$ i.e. $\bar{h}=\alpha h_{L}+(1-\alpha) h$ and 


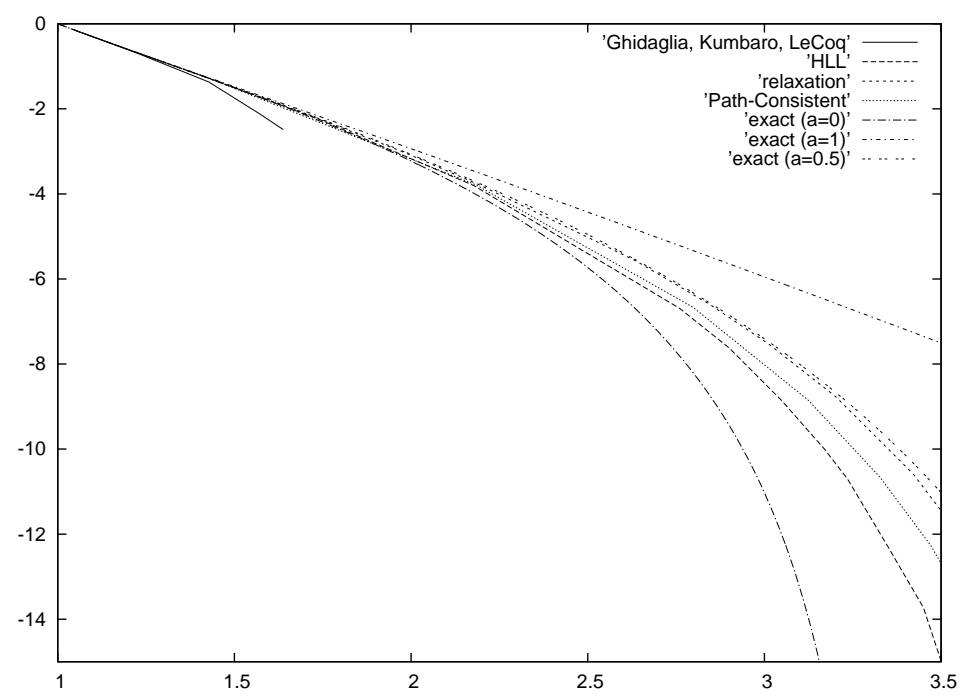

Figure 4: Hugoniot curves for a 1-shock in the Exner-Grass model: $\alpha \in[0,1]$ compared with the curves obtained by the four numerical schemes.

the obtained solutions remain inside the domain of hyperbolicity.

The first case concerns the Grass model and results can be seen on figures 4 and 5.

For shocks of very small amplitude, all results are similar. When the amplitude increases however, discrepancies can clearly be seen between the four numerical results. Interestingly, all numerical results are inside the range of path corresponding to $\alpha \in[0,1]$. It was somehow expected since they were built to be close to the straight lines path $(\alpha=0.5)$, eventually after a change of variables. However, they do not follow a curve corresponding to a constant $\alpha$ and are significantly diverging from the straight line path as the shock amplitude increases. The same remark applies for the path-consistent scheme when the change of variables is considered (the curve is not showed here for the sake of clarity). Indeed, it was already observed that path-consistent schemes do not capture the path they were built upon [9].

It is to note that the scheme of Ghidaglia, Kumbaro and Le Coq we used is unstable for large amplitude shocks in this context. In this case, the implicit version shall be used. This instability may also result from the choice of decoupling between conservative and nonconservative terms that was made here.

Finally, the (crude) HLL scheme produces comparable Hugoniot curves with respect to the other more elaborate schemes. It is obviously more diffusive but as far as the shock amplitude is concerned, it stays close to the others' predictions. The impact of the choice of different ratios between the $a$ and $b$ parameters in the relaxation scheme can be seen on figure 5. It is indeed far from being neglectable. Besides, although it is possible to adjust it so that the scheme 


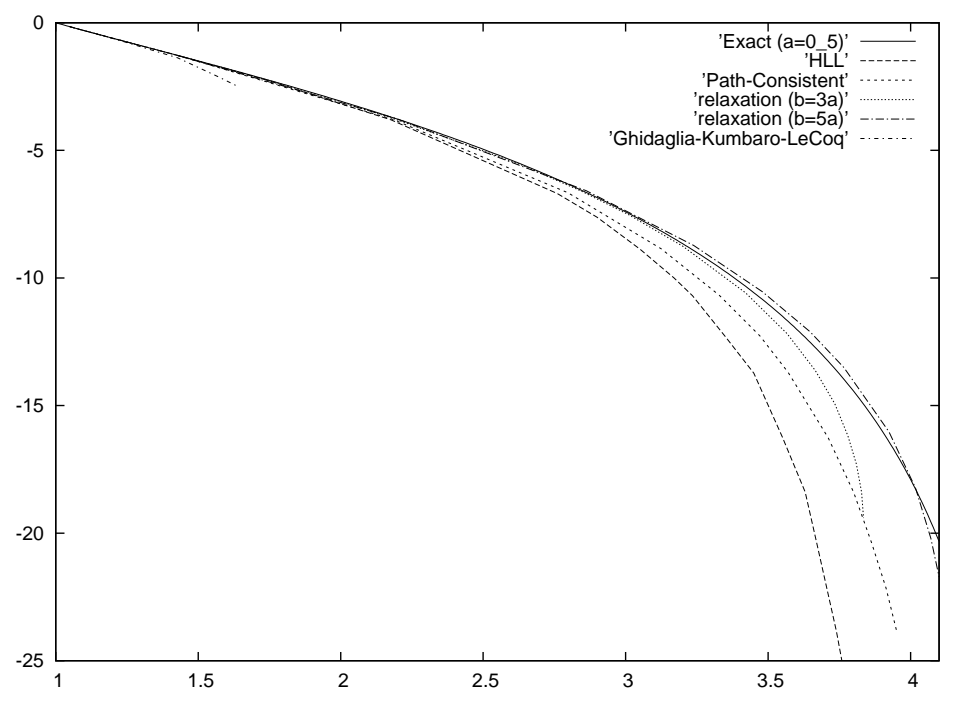

Figure 5: Hugoniot curves for a 1-shock in the Exner-Grass model: $\alpha=\frac{1}{2}$ with the curves obtained by the numerical schemes.

follows a curve very close to the straight line path, this adjustment depends on the test-case setup and imply a large numerical diffusion in the scheme since the Riemann problem is then very poorly approximated. Similar adjustments are also possible with the other schemes, as it will be seen for the HLL scheme with the Nielsen model.

It seems for these four schemes that the projection step strongly influences the path of the numerical scheme. In order to stay consistent, other techniques have to be considered (see for instance [10] in the context of nonclassical shocks). On the other hand, the influence of the time step seems to be only marginal on the results. In fact, the curves in figure 5 where all obtained with the same time step restriction ( $\Delta t$ was therefore limited by the condition on the relaxation scheme with a ratio of 5 between $a$ and $b$ ), while the curves on figure 4 were obtained using the optimal time step for each scheme. In the end, for a given scheme with a given choice of parameters, the differences were hardly visible. At most, the relative error was of the order of $10^{-3}$ with a time step 10 times smaller.

Now, the results with the Nielsen model are shown on figure 6. This time, only the HLL and path-consistent schemes were used for numerical computations. As expected, all the results are the same for $h \leq h_{\star}$ and they begin to diverge afterwards. Once again, the numerical curves do not correspond to a path defined by a constant $\alpha$ and become significantly different from the Hugoniot curves of the straight line path for large $h$. The path-consistent scheme predicts a curve 


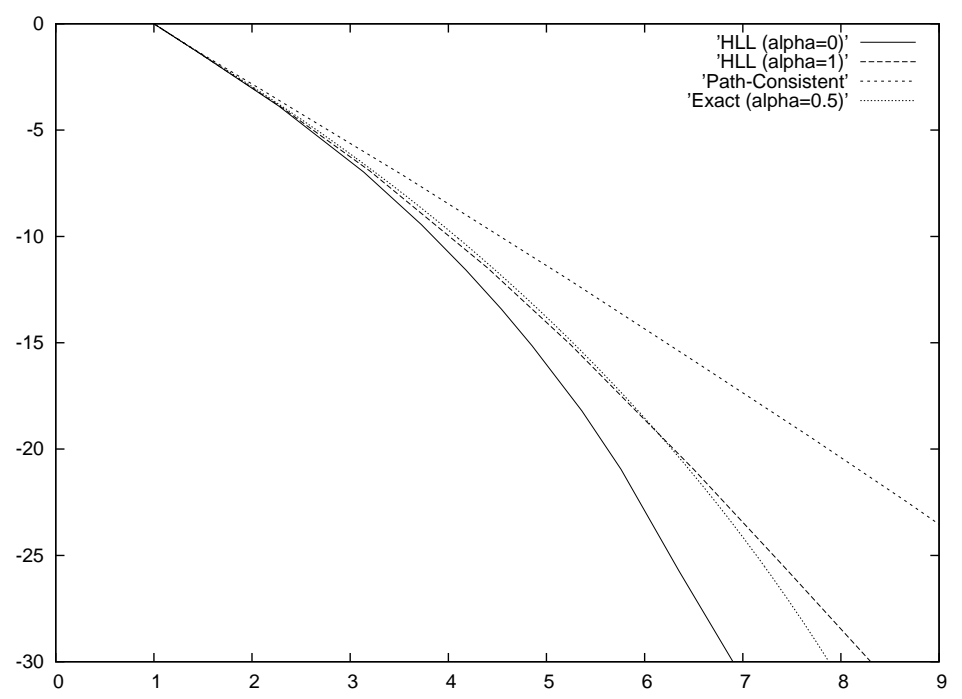

Figure 6: Hugoniot curves for a 1-shock in the Exner-Nielsen model: results for the HLL scheme with 2 definitions of $\bar{h}$, the Path-Consistent scheme and the exact solution with $\alpha=0.5$

farther to the curve associated to the path for Roe scheme. The additional discrepancies compared to the case for Grass model probably comes from the fact that the Roe mean values used in this case are those obtained for Grass model as explained in [7].

For this test, different choices of the $\bar{h}$ parameter in the HLL scheme were used. Its impact is quite important in the same way that the ratio between $a$ and $b$ impacts the relaxation scheme.

Finally, figure 7 shows the values of $h$ predicted with the HLL and pathconsistent schemes for the following Riemann problem: $h_{L}=1, u_{L}=1, z_{L}=$ 0.5 and $h_{R}=10, u_{R}=-50.58, z_{L}=1.5$. Grass model was used with $\varepsilon=6.010^{-2}$. The right state was chosen on the Hugoniot curve corresponding to a 1-shock for the straight line path.

As a consequence of the fact that the two schemes do not select the same path, the two solutions are very different from each other even though the setup used should lead results close to a single shock.

\section{Acknowledgements}

This work was partially supported by ANR GEONUM.

The authors wish to thank O. Delestre for providing his code for the relaxation model. 


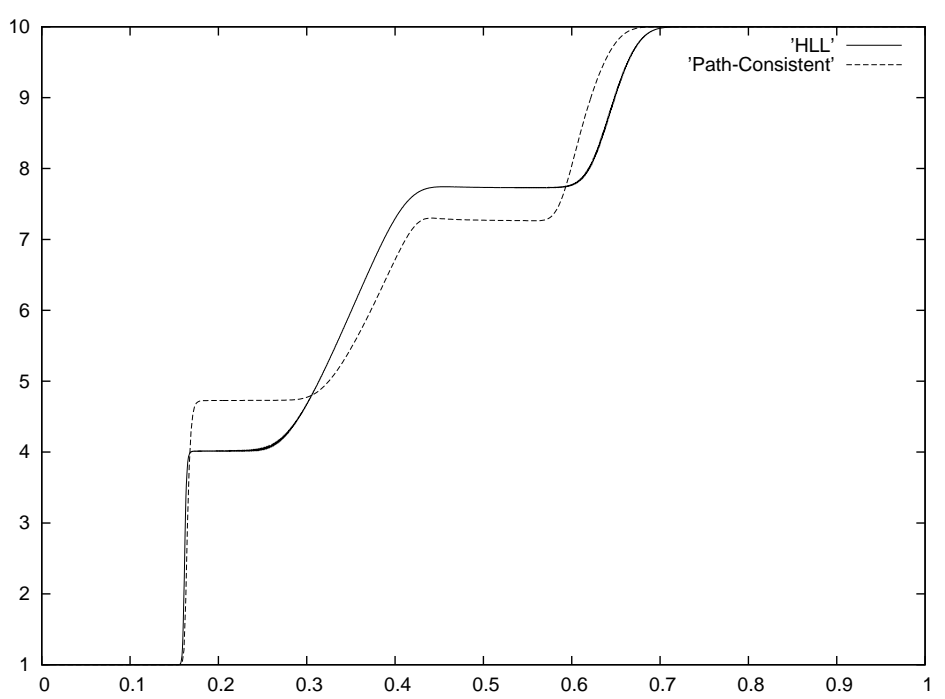

Figure 7: Comparison of $h$ predicted with the path-consistent and HLL schemes for the same initial Riemann data.

\section{References}

[1] Abgrall, R. and Karni, S., A comment on the computation of nonconservative products. J. of Comput. Phys. 2010;229:2759-2763.

[2] Audusse E., Berthon C., Chalons C., Delestre O., Goutal N., Jodeau M., Sainte-Marie J., Giesselmann J., Sadaka G., Sediment transport modelling: relaxation schemes for Saint-Venant-Exner and three layer models, ESAIM: proceedings 2012;38:78-98.

[3] Berthon C., Coquel F. and LeFloch P.G., Why many theories of shock waves are necessary: kinetic relations for non-conservative systems, Proc. Roy. Soc. Edinburgh 2012;142(01):1-37.

[4] Berthon C. and Coquel F., Nonlinear projection methods for multientropies Navier-Stokes systems, Math. Comp. 2007;76:1163-1194.

[5] Berthon C. and Coquel F., Shock layers for turbulence models, Math. Models Methods Appl. Sci. 2008;18:1443-1479.

[6] Berthon C. and Nkonga B., Multifluid numerical approximations based on a multi-pressure formulation, Comput. Fluids 2007;36:467-479.

[7] Castro Díaz M.J., Fernández-Nieto E.D. and Ferreiro A.M., Sediment transport models in Shallow Water equations and numerical approach by high order finite volume methods, Comp. \& Fluids 2008;37:299-316. 
[8] Castro Díaz M.J., Fernández-Nieto E.D., Ferreiro A.M. and Parès C., Twodimensional sediment transport models in shallow water equations. A second order finite volume approach on unstructured meshes, Comput. Methods Appl. Mech. Engrg. 2009;198:2520-2538.

[9] Castro Díaz M., LeFloch P.G., Muñoz-Ruiz M.L. and Parès C., Why many theories of shock waves are necessary: convergence error in formally pathconsistent schemes, J. Comput. Phys. 2008;227(17):8107-8129.

[10] Chalons C., Transport-Equilibrium schemes for computing nonclassical shocks. Scalar conservation laws, Numerical Methods for Partial Differential Equations 2008;24(4):1127-1147.

[11] Chalons C. and Coquel F., Euler equations with several independent pressure laws and entropy satisfying explicit projection schemes, Math. Models Methods Appl. Sci. 2006;16:1469-1504.

[12] Chalons C. and Coquel F., Numerical capture of shock solutions of nonconservative hyperbolic systems via kinetic functions, Analysis and simulation of fluid dynamics, Adv. Math. Fluid Mech., Birkhäuser, Basel 2007:45-68.

[13] Chalons C., Coquel F., Godlewski E., Raviart P.-A. and Seguin N., Godunov-type schemes for hyperbolic systems with parameter dependent source. The case of Euler system with friction. Math. Models Methods Appl. Sci. 2010;20:2109-2166.

[14] Colombeau J.F. and Le Roux A.Y., Multiplications of distributions in elasticity and hydrodynamics, J. Math. Phys. 1988;29:315.

[15] Colombeau J.F. and Le Roux A.Y and Noussair, A. and Perrot, B., Microscopic profiles of shock waves and ambiguities in multiplications of distributions, SIAM J. Numer. Anal. 1989;26:871-883.

[16] Cordier S., Le Minh H. and Morales De Luna T., Bedload transport in shallow water models: why splitting (may) fail, how hyperbolicity (can) help, Advances in Water Resources 2011;34:980-989.

[17] Dal Maso G., LeFloch P.G and Murat F., Definition and weak stability of nonconservative products, J Math Pures Appl 1995;74:483-548.

[18] Einstein H.A., The bed load function for sediment transportation in open channel ows, Technical Bulletin no. 1026, Soil Conservation Service, US Department of Agriculture 1950:1-71.

[19] Engelund F. and Fredsoe J., A sediment transport model for straight alluvial channels, Nordic Hydrol 1976;7:294-298.

[20] Exner F.M., Über die Wechselwirkung zwishen Wasser und Geschiebe in Flüssen, Sitzungsber. Akad. Wiss. Wien, Math.-Naturwiss. Kl., Abt. 2A, 1925;134:165-180. 
[21] Gallice G., Entropic Godunov-type schemes for hyperbolic systems with source term, C. R. Math. Acad. Sci. Paris 2002;334(8):713-716.

[22] Ghidaglia J.M., Kumbaro A. and Le Coq G., On the numerical solution to two fluid models via a cell centered finite volume method, Eur. J. Mech. B - Fluids 2001;20:841-867.

[23] Grass A.J., Sediments transport by waves and currents, SERC London, Cent Mar Technol report No FL29 1981.

[24] Godlewski E. and Raviart P.A., Numerical approximation of hyperbolic systems of conservation laws, Applied Mathematical Sciences 1996;118.

[25] Harten A., Lax P.D. and Van Leer B., On upstream differencing and Godunov-type schemes for hyperbolic conservation laws, SIAM Review 1983;25:35-61.

[26] Hou, T.Y. and LeFloch, P.G., Why nonconservative schemes converge to wrong solutions. Error analysis, Math. of Comput. 1994;26:497-530.

[27] LeFloch P.G. and Tzavaras A.E., Representation of weak limits and definition of nonconservative products, SIAM J. Math. Anal. 1999;30(6):13091342 .

[28] Leveque R.J., Finite volume methods for hyperbolic problems, Cambridge Texts in Applied Mathematics 2002.

[29] Meyer-Peter E. and Muller R., Formulas for bed-load transport, Proceedings of the 2nd Meeting of the International Association for Hydraulic Structures Research 1948:39-64.

[30] Nielsen P., Coastal bottom boundary layers and sediment transport. Advanced series on ocean engeneering 1992;4.

[31] Parès C., Numerical methods for nonconservative hyperbolic systems: a theoretical framework, SIAM J. Numer. Anal. 2006;44:300-321.

[32] Parès C. and Castro M.J., On the well-balance property of Roe's method for nonconservative hyperbolic systems. Applications to shallow-water systems. Math. Model. Numer. Anal. 2004;38:821-852.

[33] Raviart P.A. and Sainsaulieu L., A nonconservative hyperbolic system modelling spray dynamics. Part 1. Solution of the Riemann problem, Math. Models Methods in App. Sci. 1995;5(3):297-333.

[34] Roe P.L., Approximate Riemann solvers, parameter vectors and difference schemes, J. Comput. Phys. 1981;43:237-263.

[35] Volpert A.I., The space BV and quasilinear equations, Math. USSR Sbornik $1967 ; 73(115): 225-267$. 